\title{
Reconstruction of the solar spectral UV irradiance for nowcasting of the middle atmosphere state on the basis of LYRA measurements
}

\author{
T. Egorova ${ }^{1}$, E. Rozanov ${ }^{1,2}$, J.-F. Hochedez ${ }^{3}$, and W. Schmutz ${ }^{1}$ \\ ${ }^{1}$ Physikalisch-Meteorologisches Observatorium Davos and World Radiation Center, Dorfstrasse 33, \\ 7260 Davos Dorf, Switzerland \\ ${ }^{2}$ Institute for Atmospheric and Climate Science, ETH Zürich, Universitätsstr. 16, 8092 Zürich, Switzerland \\ ${ }^{3}$ Royal Observatory of Belgium, Circular Avenue 3, 1180 Brussels, Belgium
}

Received: 3 January 2008 - Published in Atmos. Chem. Phys. Discuss.: 28 February 2008

Revised: 15 May 2008 - Accepted: 19 May 2008 - Published: 12 June 2008

\begin{abstract}
The LYRA instrument onboard ESA PROBA2 satellite will provide 6-hourly solar irradiance at the Lymanalpha $(121.6 \mathrm{~nm})$ and the Herzberg continuum ( 200$220 \mathrm{~nm}$ wavelength range). Because the nowcasting of the neutral and ionic state of the middle atmosphere requires the solar irradiance for the wide spectral range $(120-680 \mathrm{~nm})$ we have developed the statistical tool for the reconstruction of the full spectrum from the LYRA measurements. The accuracy of the reconstructed irradiance has been evaluated with 1-D transient radiative-convective model with neutral and ion chemistry using the daily solar spectral irradiance measured with SUSIM and SOLSTICE instruments onboard UARS satellite. We compared the results of transient 1-year long model simulations for 2000 driven by the observed and reconstructed solar irradiance and showed that the reconstruction of the full spectrum using linear regression equation based on the solar irradiance in two LYRA channels can be successfully used for nowcasting of the middle atmosphere. We have also identified conditions when the proposed approach does not yield spectral reconstruction with sufficient accuracy.
\end{abstract}

\section{Introduction}

The nowcasting and short-term forecasting of the space weather driven by the different observational data gained recently a lot of attention, because the Solar activity variations are able to induce substantial changes in the Earth environment, which turn out to be important for the space operations (Wilkinson, 1994), radio-wave propagation, GPS functioning and many other aspects of the mankind activity (Jansen

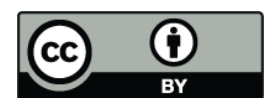

Correspondence to: E. Rozanov

(e.rozanov@pmodwrc.ch) et al., 2000). Among other elements of the space weather it is of interest to understand and predict the response of the neutral and charged species in the middle atmosphere to the variability of the solar activity. An important aspect of this issue is an evaluation and prediction of the response of the middle atmosphere to the solar ultraviolet irradiance variability, which defines the behavior of the species and temperature in the middle atmosphere via the perturbation of the photolysis, photoionization and heating rates (Brasseur and Solomon, 2005). On the other hand the response of the middle atmosphere to the solar variability can be modulated by complicated non-linear dynamics, advective and turbulent transport of heat and species. Therefore, nowcasting of the middle atmosphere state requires application of appropriate models and detailed knowledge of the solar spectral irradiance in real time.

High quality solar UV irradiance observation data are available from a variety of past (Floyd et al., 1998; Rottman et al., 1993) and ongoing (Rottman et al., 2006) satellite experiments. The solar irradiance measurements by the SORCE experiment (http://earthobservatory.nasa.gov/ Library/SORCE), if available in real-time, could provide a solid basis for the nowcasting of the neutral and ion composition of the stratosphere and mesosphere. More near real-time solar irradiance data will be available in the future. The LYRA instrument onboard PROBA-2 satellite will provide the solar irradiance for several wavelengths important for the middle atmosphere in real time (Hochedez et al., 2006). LYRA will specifically monitor the solar irradiation in four wavelength bands that are essential for the Sun-Earth connection: Lyman-alpha (115-125 nm), Herzberg (200$220 \mathrm{~nm})$, Zirconium $(1-20 \mathrm{~nm})$ and Aluminium $(17-70 \mathrm{~nm})$ including $\mathrm{He}$ II at $30.4 \mathrm{~nm}$. It will be the first space assessment of the pioneering UV detectors from the BOLD program (http://bold.oma.be), which make use of wideband-gap materials instead of silicon. Another instrument

Published by Copernicus Publications on behalf of the European Geosciences Union. 
PREMOS on the French satellite PICARD (Thuillier et al., 2006) will monitor the solar irradiance in two UV channels at $270 \mathrm{~nm}$ and in the Herzberg band, and in one visual channel at $535 \mathrm{~nm}$ and one near IR channel at $782 \mathrm{~nm}$. The LYRA and PREMOS observations can be used as input data for global climate-chemistry-ionosphere models in order to evaluate the response of the terrestrial atmosphere to the solar UV irradiance variability. These results are indispensable for space whether and global climate applications.

However, while it is necessary to have full solar spectrum to drive a nowcasting, the observational data by LYRA and PREMOS cover only several spectral intervals. Therefore, to apply LYRA and PREMOS data it is necessary to reconstruct the solar UV spectrum. The attempts to reconstruct solar irradiance have been made by several groups (e.g. by Krivova et al., 2006; Dudok de Wit et al., 2005) on the basis of statistical approaches. Krivova et al. (2006) applied for the solar UV irradiance reconstruction a semi-empirical model assuming all irradiance variations to be due to the evolution of the solar surface magnetic features and showed that this approach performs well for the wavelengths longer than $\sim 220 \mathrm{~nm}$. For the shorter wavelengths Krivova et al. (2006) used regression equations based on the correlation with the measured solar UV irradiance at 220-240 nm. Dudok de Wit et al. (2005) used statistical approach based on the singular value decomposition analysis and showed that all spectral lines can be conveniently represented on a 2-D map according to the similarities in their temporal evolution. They showed that a subset of 5 to 8 of these lines is sufficient for reconstructing the spectrum with a relative error below $0.25 \%$. These efforts suggested that the full spectrum could be successfully retrieved from the limited number of spectral channels.

The main goal of the paper is to show that the solar UV irradiance can be reconstructed with a correlation better than 0.8 and root mean square (RMS) difference smaller than $40 \%$ from the LYRA measurements using regression analysis. Because LYRA is not yet operational we use UARS SUSIM and SOLSTICE daily solar UV irradiance for the year 2000. From these data it is possible to calculate the solar irradiance for the LYRA channels and apply it to reconstruct the full solar UV spectrum using linear regression technique, which can be then evaluated against the reference solar UV spectrum. Because we are interested mostly in the accuracy of the final product (i.e., state of the middle atmosphere) it is worthwhile to apply a goal-oriented approach and compare the temperature and species distributions simulated with a model driven by the reference and reconstructed solar UV irradiance. This approach allows us to filter out the difference in the solar irradiance, which is not really important for the simulation of the atmospheric state. It is also of interest to look at some other proxies and estimate their potential for the UV irradiance reconstruction. In case of success they can be considered as auxiliary input for the regression model. In this paper we considered the following additional proxies: solar UV irradiance at $205 \mathrm{~nm}$; total solar irradiance; solar radio flux at $10.7 \mathrm{~cm}$; neutron monitor data.

The structure of the paper is the following. The applied 1-D model is described in Sect. 2. The results of the regression analysis are presented in Sect. 3. In Sect. 4 we discuss the results of the goal-oriented evaluation. Conclusions and outlook are presented in Sect. 5.

\section{Model description}

For this study we applied a 1-D radiative-convective model with interactive photochemistry (RCMP) described by Rozanov et al. (2002) and extended to take into account chemistry of the charged species in the middle atmosphere. The 1-D RCMP consists of the radiation, chemistry, convective adjustment and vertical diffusion modules. The model spans the atmosphere from the ground to $100 \mathrm{~km}$. For the calculation of long wave cooling rates we have applied the radiation scheme of Frolkis and Rozanov (1992) for the atmosphere below $1 \mathrm{hPa}$ and that of Fomichev et al. (1998) to treat non-LTE processes in the mesosphere. For the calculation of the photolysis and solar heating rates we use also modified scheme of Frolkis and Rozanov (1992). The heating and photolysis rate calculation scheme spans the spectral region from 120 to $750 \mathrm{~nm}$. The entire spectral region is divided into 73 spectral intervals.

The photochemical part of the model includes neutral and ion chemistry. It calculates the distribution of 43 neutral chemical species of the oxygen, nitrogen, hydrogen, carbon, chlorine and bromine groups with 173 reactions. The ion chemistry includes 48 ions (31 Positive and 17 negative) with 278 reactions. The main positive ions are $\mathrm{O}^{+}$, $\mathrm{O}_{2}^{+}, \mathrm{N}_{2}^{+}, \mathrm{NO}^{+}$, and $\mathrm{O}_{4}^{+}$. The main negative ions are $\mathrm{O}^{-}$, $\mathrm{O}_{2}^{-}, \mathrm{O}^{-}, \mathrm{O}_{4}^{-}, \mathrm{OH}^{-}, \mathrm{CO}_{3}^{-}, \mathrm{CO}_{4}^{-}, \mathrm{NO}_{2}^{-}, \mathrm{NO}^{-}$, and $\mathrm{HCO}_{3}^{-}$. It takes into account proton hydrates $\mathrm{H}^{+}\left(\mathrm{H}_{2} \mathrm{O}\right)_{n}, \mathrm{n}=1 \ldots 7$, $\mathrm{NO}^{+}$hydrates $\mathrm{NO}^{+}(\mathrm{H} 2 \mathrm{O})_{n}, \mathrm{n}=1,2,3, \mathrm{NO}^{+}\left(\mathrm{H}_{2} \mathrm{O}\right)_{n} \mathrm{~N}_{2}$ and $\mathrm{NO}^{+}\left(\mathrm{H}_{2} \mathrm{O}\right)_{n} \mathrm{CO}_{2}$, where $\mathrm{n}=1,2$. Other ion clusters included are $\mathrm{NO}^{+}\left(\mathrm{N}_{2}\right), \mathrm{NO}^{+}\left(\mathrm{CO}_{2}\right), \mathrm{O}_{2}^{+}\left(\mathrm{H}_{2} \mathrm{O}\right)$, and $\mathrm{H}_{3} \mathrm{O}^{+}(\mathrm{OH})$. The sources of ionization are galactic cosmic rays (GCR), energetic electron precipitation (EEP), solar radiation, and solar proton events (SPEs). For GCR we utilize the parameterization by Heaps (1978), for SPEs we use the database of solar proton fluxes (http://www.geo.fu-berlin.de/en/met/ag/ strat/research/SOLARIS/Input_data/index.html). The chemical solver utilizes pure implicit iterative Newton-Raphson scheme (Rozanov et al., 1999). The reaction coefficients are taken from Sander et al. (2003) for neutral atmosphere and from Koop (1996) and Kazil (2002) for ionosphere. The convective adjustment is treated according to Egorova et al. (1997) and vertical transport of species is calculated using typical annually mean values of the diffusion coefficient. 
a)

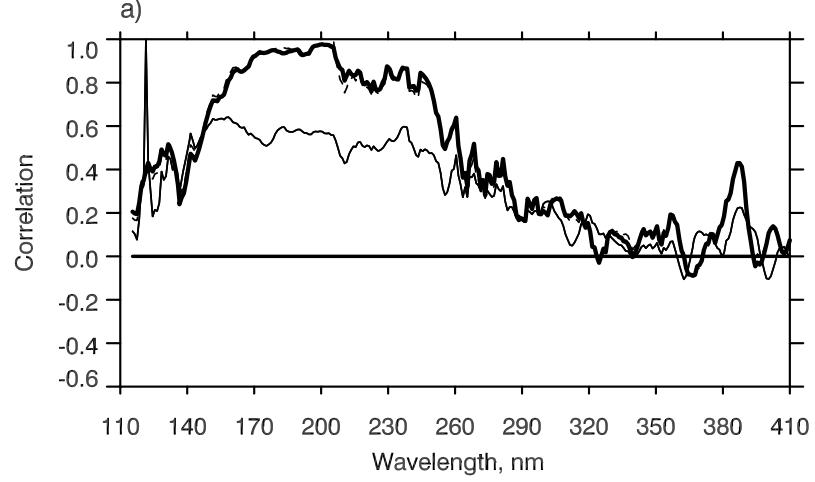

b)

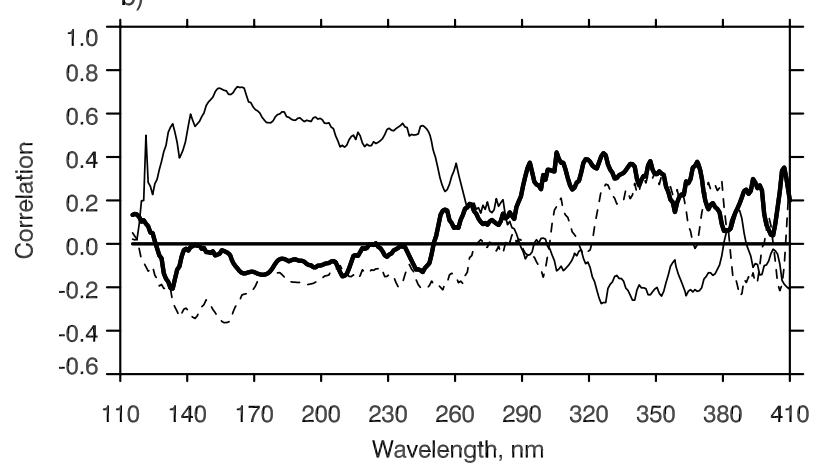

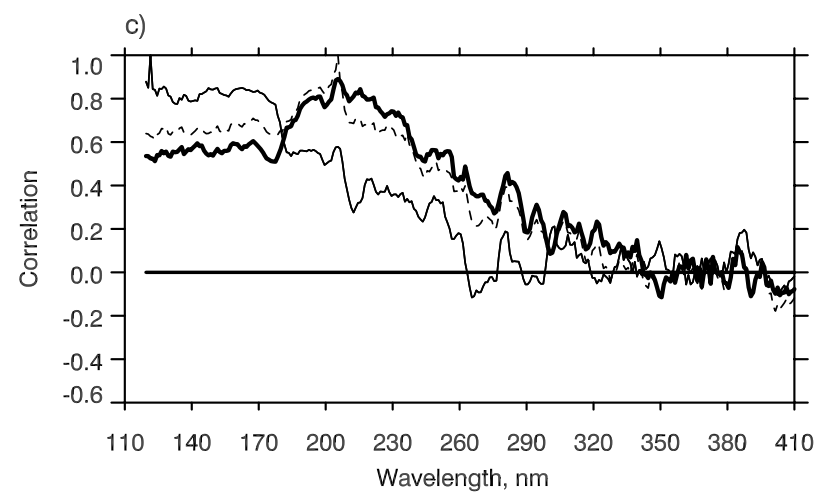

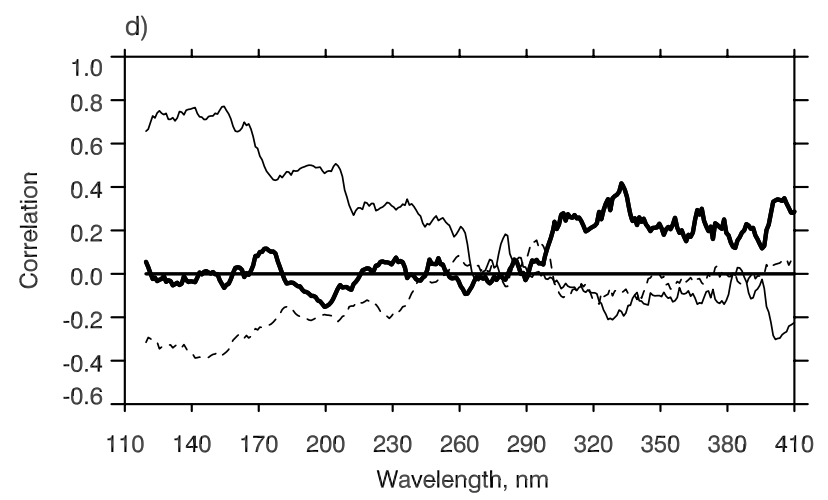

Fig. 1. The correlation of the spectral SUSIM (a,b) and SOLSTICE (c,d) solar irradiance with different proxies: Ly- $\alpha$ (thin line), $205 \mathrm{~nm}$ (dashed line), Herz (thick line) (a, c) and TSI (thick line), F10.7(thin line), and NMD (dashed line) (b, d) for the year 2000.

\section{Regression analysis}

As the input for the model we use spectral solar irradiance obtained with SUSIM and SOLSTICE instruments on board UARS satellite (Woods et al., 1996), which are publicly available from the NASA ftp site: disc.sci.gsfc.nasa.gov. For the present study we used daily average data for 2000 , which corresponds to the solar maximum.

First of all we calculated the correlation coefficients between the observed with SUSIM and SOLSTICE daily solar spectral UV irradiance for all wavelengths and considered proxies. Ly- $\alpha$ proxy is the solar irradiance in Lyman-alpha line $(121-122 \mathrm{~nm}) .205 \mathrm{~nm}$ proxy is the solar irradiance in the 204-205 nm spectral bin. HERZ proxy is the solar irradiance in the Herzberg channel of the LYRA instrument. The latter has been constructed from the LYRA Herzberg continuum channel taking into account responsivity of LYRA instrument detectors provided by I. Dammasch (Royal Observatory of Belgium). These three proxies have been calculated from the both SUSIM and SOLSTICE data sets. TSI proxy is the total solar irradiance from the VIRGO experiment (ftp://ftp.pmodwrc.ch/pub/virgo/data). F10.7 proxy is the solar radio flux at $10.7 \mathrm{~cm}$ (www.ngdc.noaa.gov/stp/SOLAR/ ftpsolarradio.html) and NMD proxy is the neutron monitor data (www.ngdc.noaa.gov/stp/SOLAR/ftpcosmicrays.html).
All proxies were calculated on daily basis corresponding to the SUSIM and SOLSTICE measurements. The smoothed (using boxcar average over $3 \mathrm{~nm}$ window) correlation coefficients are shown in the Fig. 1.

The correlation coefficients for HERZ and $205 \mathrm{~nm}$ proxies have almost identical behavior. Their correlation with SUSIM spectral irradiance is rather low for the wavelength shorter than $\sim 160 \mathrm{~nm}$, but it becomes quite high $(>0.8)$ between $160 \mathrm{~nm}$ and $250 \mathrm{~nm}$. For the longer wavelengths the correlation is steadily decreasing. For the SOLSTICE data the correlations differ from the previous case mainly below $160 \mathrm{~nm}$. In this area the correlation with $205 \mathrm{~nm}$ is much higher $(\sim 0.5)$ than for SUSIM data. Ly- $\alpha$ proxy also works much better in this spectral region for SOLSTICE data, while for longer wavelengths $(>170 \mathrm{~nm})$ this proxy has very week correlation with spectral irradiance. It is also evident from the Fig. 1 (a, c) that the spectral solar irradiance in the spectral regions shorter and longer $\sim 260 \mathrm{~nm}$ are not well connected at least for the applied considered daily time scale. This fact was also discussed previously by Unruh et al. (2008). The correlation of the solar spectral irradiance with F10.7 proxy is higher for the short-wave bands (120$250 \mathrm{~nm}$ ), but even in this case it hardly reaches 0.5 . The TSI and NMD proxies have almost no correlation with solar irradiance between 120 and $270 \mathrm{~nm}$, while for the rest of the 
UV spectrum TSI has a better correlation $(\sim 0.4)$ than the other proxies. In general, the reconstructions of the solar irradiance using Ly- $\alpha, 205 \mathrm{~nm}$ and HERZ proxies are more accurate than the reconstructions based on the F10.7 proxy. According to our analysis the TSI and NMD proxies do not allow realistic reconstruction of the solar UV irradiance variability. The best correlation can be reached using a combination of the HERZ and Ly- $\alpha$ proxies. These two quantities will be measured by LYRA instrument and hereafter this combined proxy will be called LYRA-P. For the LYRA$P$ proxy the daily spectral UV irradiance is calculated from the HERZ proxy for all wavelengths except Ly- $\alpha$ line, where the measured data are directly applied.

The difference in the results of the correlation analysis for the spectral range $120-170 \mathrm{~nm}$ with UARS SUSIM and UARS SOLSTICE data sets most probably has instrumental nature. This issue was discussed in detail by Woods et al. (1996). They pointed out that the sensor degradation and instrumental noise can deteriorate the quality of the data in these spectral intervals, which leads in our case to severely different correlations for two considered UARS instruments.

Using proxies described above we have reconstructed solar UV irradiance in the spectral interval $120-750 \mathrm{~nm}$ required by the model applying linear regression analysis. We use the following regression equation for the reconstruction:

$F_{\lambda}^{i}=A_{\lambda}+B_{\lambda} \times P^{i}$

where $\boldsymbol{F}$ is solar spectral UV irradiance; $\lambda$ is wavelength; $\boldsymbol{i}$ is a day number; $\boldsymbol{P}$ is solar irradiance proxy: $\mathrm{Ly}-\alpha, 205 \mathrm{~nm}$, HERZ, TSI, F10.7, or NMD; $\boldsymbol{A}$ and $\boldsymbol{B}$ are wavelength dependent regression coefficients. For the combined LYRA-P proxy we calculate the solar irradiance from Eq. (1) for all wavelengths using LYRA Herzberg channel irradiance as $\boldsymbol{P}$, except Ly- $\alpha$ line, where the observed values are used.

\section{Accuracy evaluation}

From the correlation analysis presented in the Sect. 3 it is hard to conclude how accurate would be the simulation of the atmospheric state driven by the reconstructed solar irradiance, because the solar irradiance at different wavelengths has different influence on the atmospheric state (e.g., Rozanov et al., 2002) and it could well be that a substantial error in the solar irradiance would not affect some of the simulated atmospheric quantities. Therefore, the accuracy of the reconstruction should be evaluated by the comparison of the atmospheric state variability simulated with a model driven by the observed and reconstructed daily spectral solar UV irradiance. We performed several 1-year long runs with the 1-D RCMP described in Sect. 2. Two reference runs were driven by the observed solar irradiance from UARS SUSIM and UARS SOLSTICE instruments. The other twelve runs have been performed with the solar irradiance reconstructed from different proxies: Ly- $\alpha, 205 \mathrm{~nm}$, LYRA-P, TSI, F10.7, and NMD for the both instruments.

To characterize the accuracy of the reconstruction we calculated the correlation coefficients and relative RMS differences between the daily temperature, ozone, hydroxyl and total positive ions concentration simulated with the observed and reconstructed solar spectral UV irradiance. The analysis of these quantities shows which proxy is the most accurate for any particular altitude. The vertical profiles of the correlation coefficient and RMS differences for the total positive ions concentration are shown in Fig. 2. The ionization rates for our model are defined mostly by the photoionization of NO and by galactic cosmic rays (GCR). The photoionization of NO occurs by the soft solar UV irradiance (Brasseur and Solomon, 2005), therefore its concentration in the mesosphere should be well defined there by the solar irradiance in Lyman- $\alpha$. In the stratosphere, where the influence of GCR is noticeable the total number of positive ions also depends on the atmospheric temperature and neutral species. Due to the former process (NO photoionization) the accuracy of the solar irradiance reconstruction using Ly- $\alpha$ and LYRA-P proxies is very good in the mesosphere above $60 \mathrm{~km}$. The correlation coefficients are close to 1.0 and RMS difference is less than $10 \%$. Between 50 and $60 \mathrm{~km} 205 \mathrm{~nm}$ and LYRA-P proxies provide the best accuracy. This confirms the importance of the temperature and neutral species, which are defined (see below) for the most part of the stratosphere by the solar irradiance in Herzberg continuum.

The vertical profiles of the correlation coefficient and RMS differences for the hydroxyl are shown in Fig. 3. Hydroxyl behavior is very complicated and is defined by multitude of physical and chemical processes in the atmosphere (e.g., Brasseur and Solomon, 2005). In the mesosphere, however, the dominant source of hydroxyl is photolysis of the water vapor in Lyman- $\alpha$ line; therefore above $60 \mathrm{~km}$ the best accuracy is provided by Ly- $\alpha$ and LYRA-P proxies. The correlation coefficients are close to 1.0 and RMS difference does not exceed 20\%. From 20 to $60 \mathrm{~km}$ the production of hydroxyl depends on the ozone and excited atomic oxygen concentrations, which are better represented by $205 \mathrm{~nm}$ and LYRA-P proxies. For these altitudes the correlation is still high $(\sim 0.5)$ for SUSIM data set, but for the SOLSTICE reasonable accuracy cannot be achieved. The RMS differences are large $(\sim 100 \%)$ below $40 \mathrm{~km}$ for both data sets, which means that none of the considered proxies are able to provide satisfactory accuracy for the hydroxyl between 20 and $40 \mathrm{~km}$.

The vertical profiles of the correlation coefficient and RMS differences for the ozone are shown in Fig. 4. The ozone sensitivity to the solar spectral UV irradiance variability has been considered in many publications (see Brasseur and Solomon, 2005 and references therein). Rozanov et al. (2002) found out that an increase of solar UV irradiance in the spectral region 170-240 nm leads to the ozone enhancement between 20 and $90 \mathrm{~km}$ due to additional molecular 

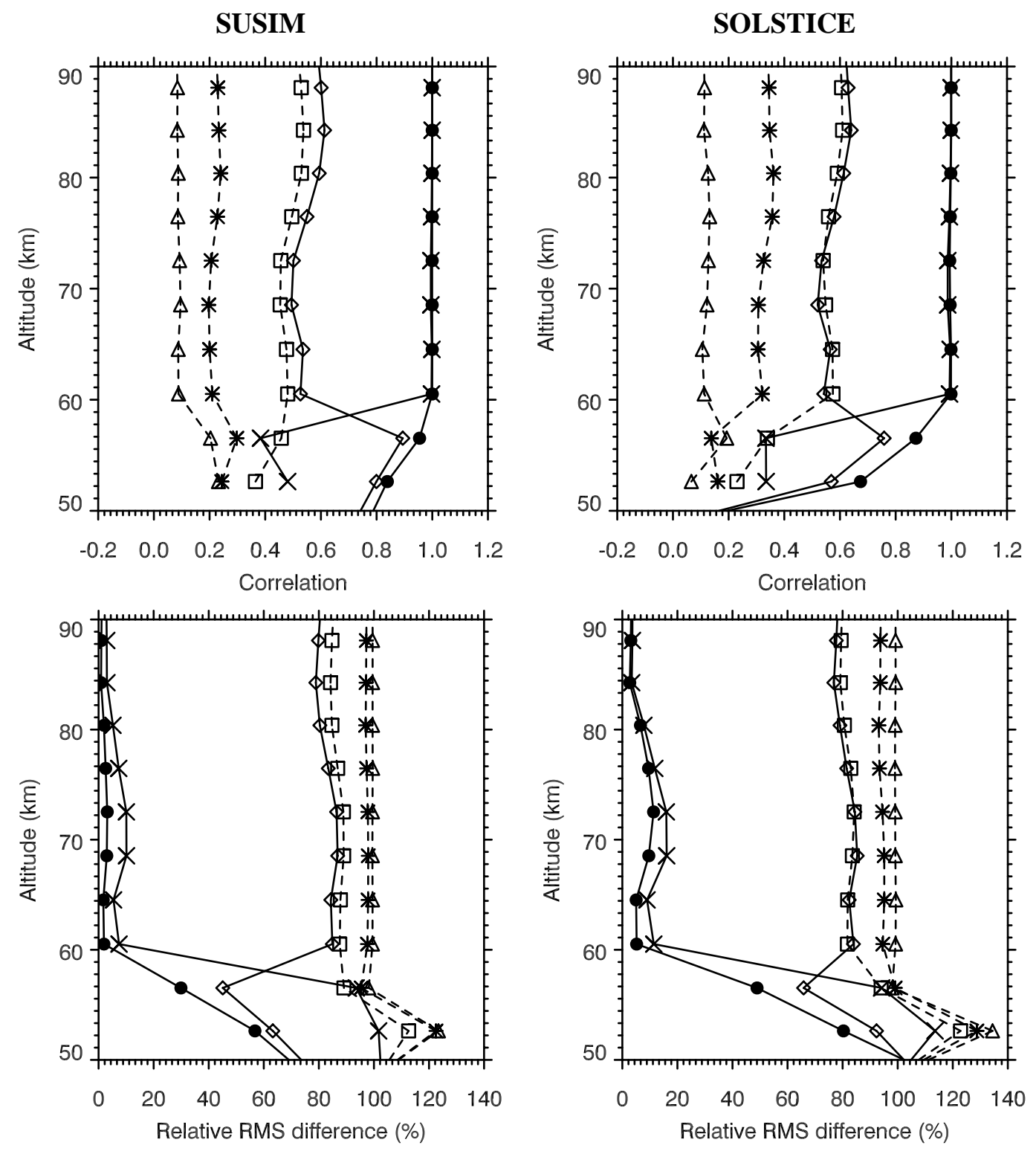

Fig. 2. Positive ions: Vertical profiles of the correlation (upper row) and relative RMS difference (lower row) of daily variations of the total positive ions for SUSIM (left column) and SOLSTICE (right column) with different proxies: Ly- $\alpha$ (solid line, crosses), $205 \mathrm{~nm}$ (solid line, diamonds), LYRA-P (solid line, filled circles), TSI (dashed line, triangles), F10.7(dashed line, squares), and NMD (dashed line, asterisks).

oxygen photolysis and subsequent increase of the ozone production, while an increase of the solar irradiance in Hartley band $(240-300 \mathrm{~nm})$ results in the ozone depletion from 40 to $70 \mathrm{~km}$ due to elevated ozone destruction rates by the enhanced ozone photolysis. Therefore the accuracy of the ozone simulation depends on the accuracy of the solar UV reconstruction in rather wide spectral range and also on the variability of the applied solar UV irradiance. In case of SUSIM data the accuracy of the ozone prediction is very high with LYRA-P and $205 \mathrm{~nm}$ proxies below $60 \mathrm{~km}$ and LYRA-P and Ly- $\alpha$ proxies above $60 \mathrm{~km}$. For the SOLSTICE data the ozone simulation has much lower accuracy in the $40-65 \mathrm{~km}$ level. This effect is caused by the fact that the variability of the solar UV irradiance in Hartley band in SOLSTICE data is higher than in SUSIM data (e.g., Rozanov et al., 2008). As it is clear from Fig. 1 our proxies do not properly reproduce the solar irradiance in the Hartley band, which results in unaccounted ozone variability in the case of the SOLSTICE data. This effect is not so pronounced for the SUSIM data because the variability of the solar irradiance in Hartley band is much smaller.

The vertical profiles of the correlation coefficient and RMS differences for the temperature are shown in Fig. 5. The temperature sensitivity to the solar spectral UV irradiance 
SUSIM
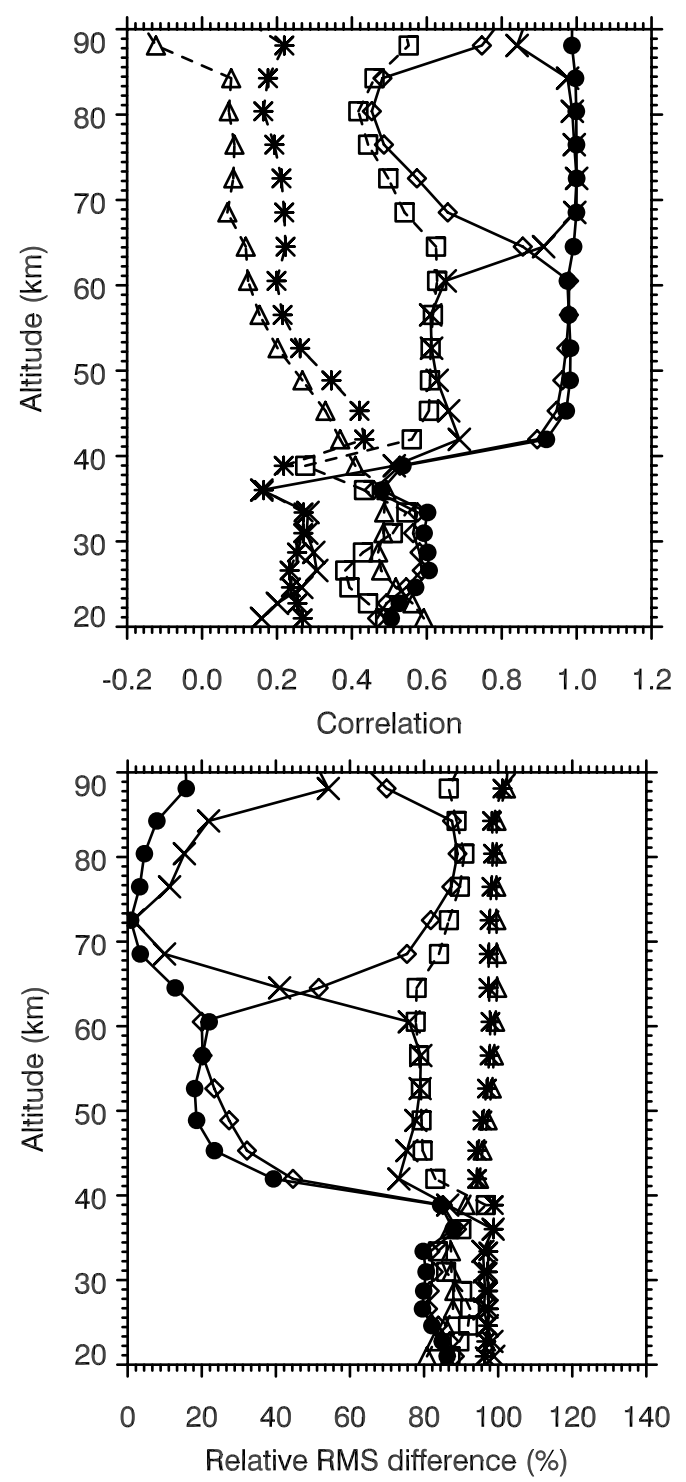

SOLSTICE
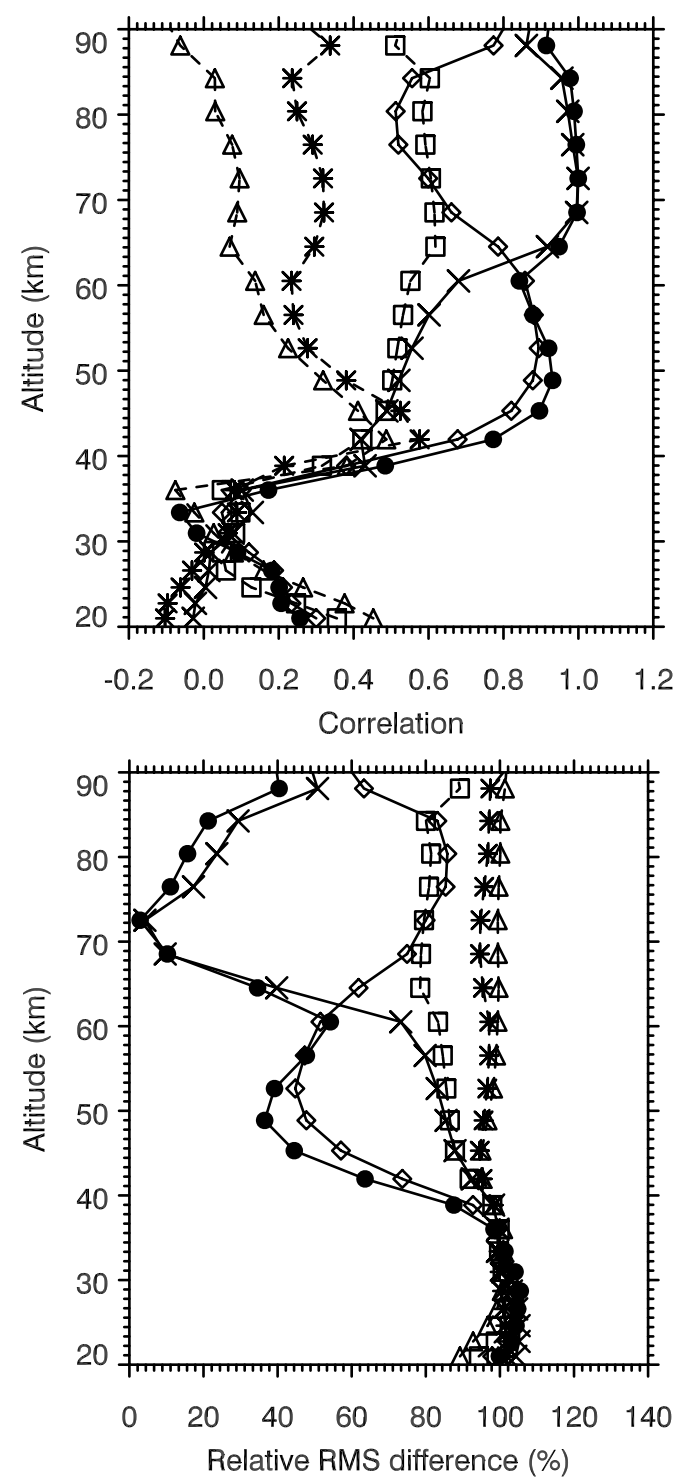

Fig. 3. Hydroxyl: Vertical profiles of the correlation (upper row) and relative RMS difference (lower row) of daily variations of the hydroxyl for SUSIM (left column) and SOLSTICE (right column) with different proxies: Ly- $\alpha$ (solid line, crosses), $205 \mathrm{~nm}$ (solid line, diamonds), LYRA-P (solid line, filled circles), TSI (dashed line, triangles), F10.7(dashed line, squares), and NMD (dashed line, asterisks).

variability has been analyzed by Rozanov et al. (2002). They found out that the observed enhancement of solar UV irradiance results in the warming of the entire stratosphere due to absorption of additional energy by ozone and oxygen. The oxygen Schumann-Runge band is responsible for the heating of the mesosphere, while the ozone absorption in Herzberg continuum and part of the ozone Hartley band (260-300 nm) warms up the stratosphere. It should be noted that the contribution of the Hartley band is slightly smaller in comparison with Herzberg continuum, and the absorption in Lyman- $\alpha$ is noticeable only in the upper mesosphere.
Because the solar irradiance in Schumann-Runge band and Herzberg continuum is well correlated with LYRA-P and $205 \mathrm{~nm}$ proxies, the accuracy of the temperature simulation is rather high especially above $40 \mathrm{~km}$. Below $40 \mathrm{~km}$ the influence of Hartley band is noticeable and the accuracy of the temperature simulations drops down. The results also demonstrated that the other proxies (including F10.7) do not provide sufficient accuracy for the simulation of all considered quantities. 


\section{SUSIM}
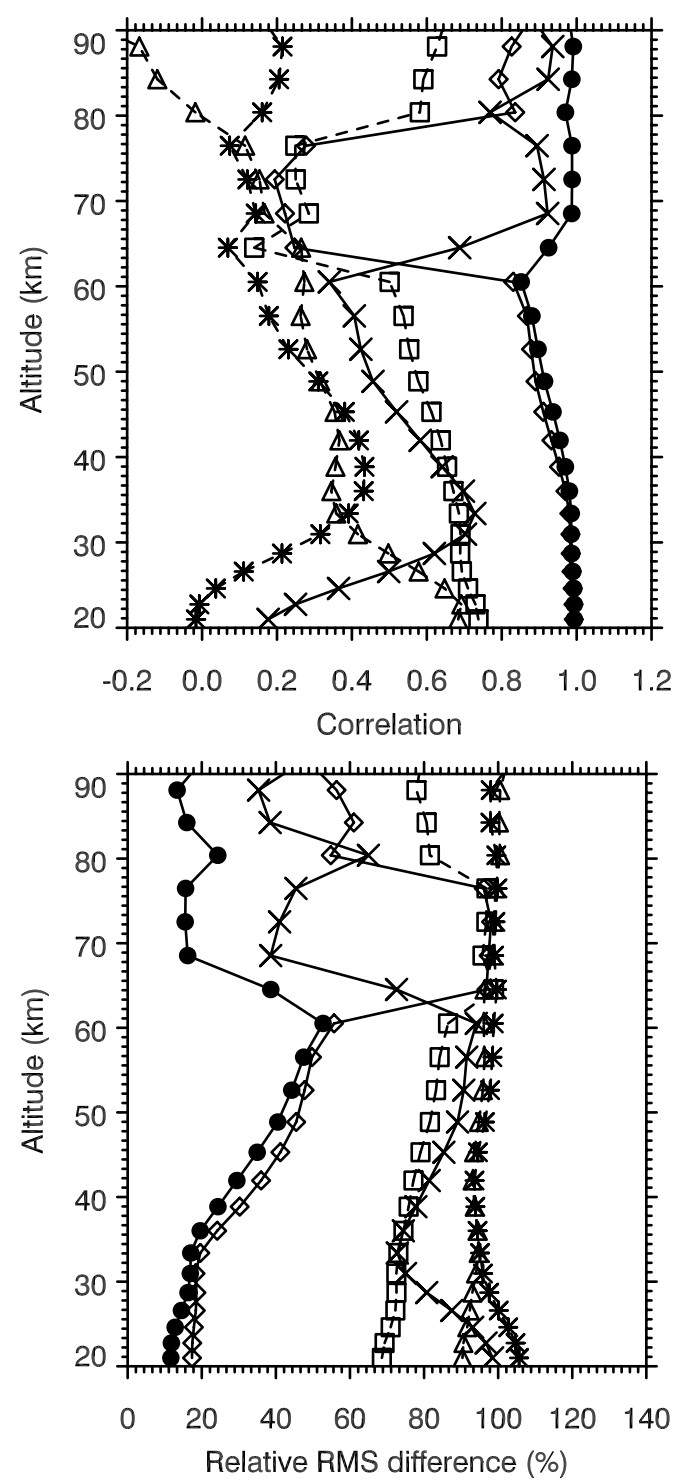

SOLSTICE
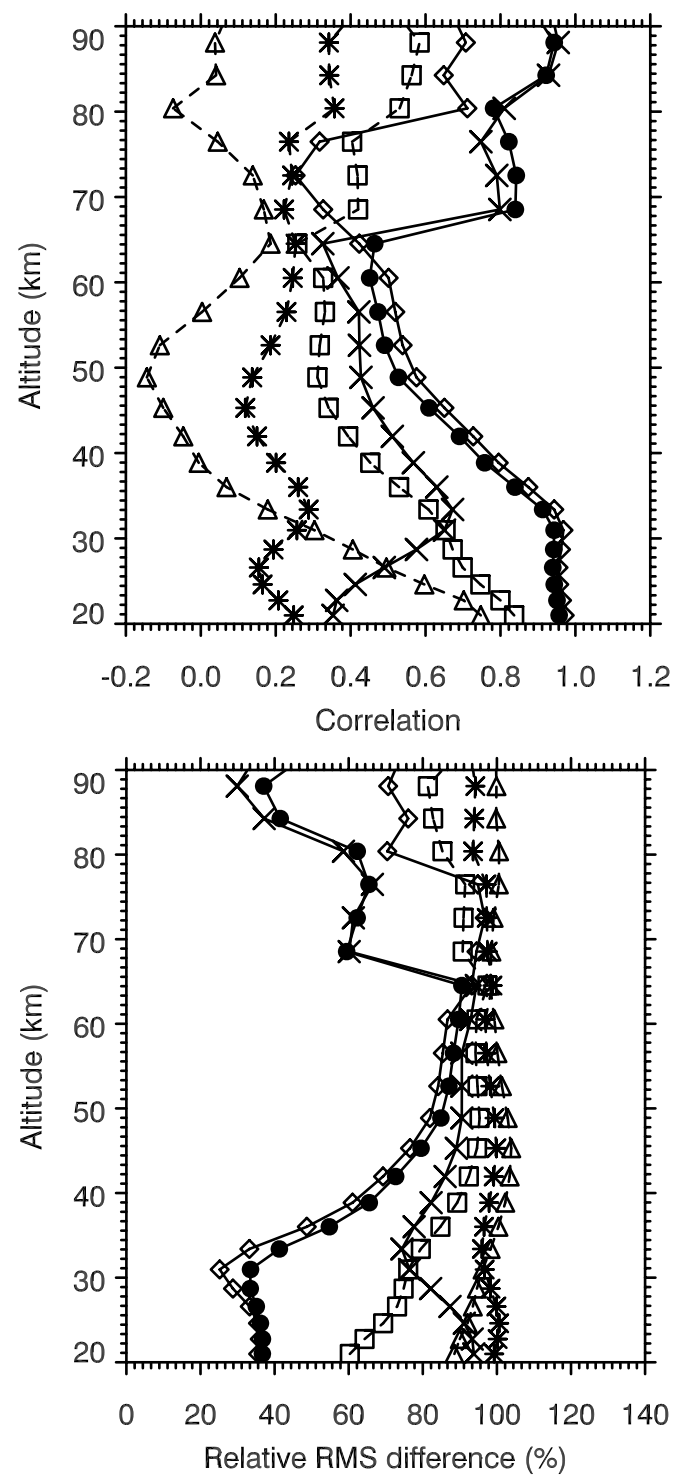

Fig. 4. Ozone: Vertical profiles of the correlation (upper row) and relative RMS difference (lower row) of daily variations of the ozone for SUSIM (left column) and SOLSTICE (right column) with different proxies: Ly- $\alpha$ (solid line, crosses), $205 \mathrm{~nm}$ (solid line, diamonds), LYRA-P (solid line, filled circles), TSI (dashed line, triangles), F10.7(dashed line, squares), and NMD (dashed line, asterisks).

\section{Conclusions}

In this paper we evaluate the applicability of the data from the future LYRA experiment onboard ESA PROBA2 satellite for the nowcasting of the middle atmosphere state. The LYRA instrument onboard ESA PROBA2 satellite will provide 6hourly solar irradiance at the Lyman-alpha $(121.6 \mathrm{~nm})$ and the Herzberg continuum ( $\sim 200-220 \mathrm{~nm}$ wavelength range). To reconstruct the solar irradiance for the wide spectral range (120-680 nm) needed for nowcasting models we have applied the statistical tool based on the correlation of the solar spectral UV irradiance with different proxies using the daily solar spectral irradiance measured with SUSIM and SOLSTICE instruments onboard UARS satellite. To estimate the accuracy of the reconstructed solar irradiance we have applied 1-D transient radiative-convective model with neutral and ion chemistry. We compared the results of transient 1year long model simulations for the year 2000 driven by the observed and reconstructed solar irradiance and showed that the reconstruction of the full spectrum using linear regression equation based on the solar irradiance in two LYRA channels can be successfully used for nowcasting of the 


\section{SUSIM}
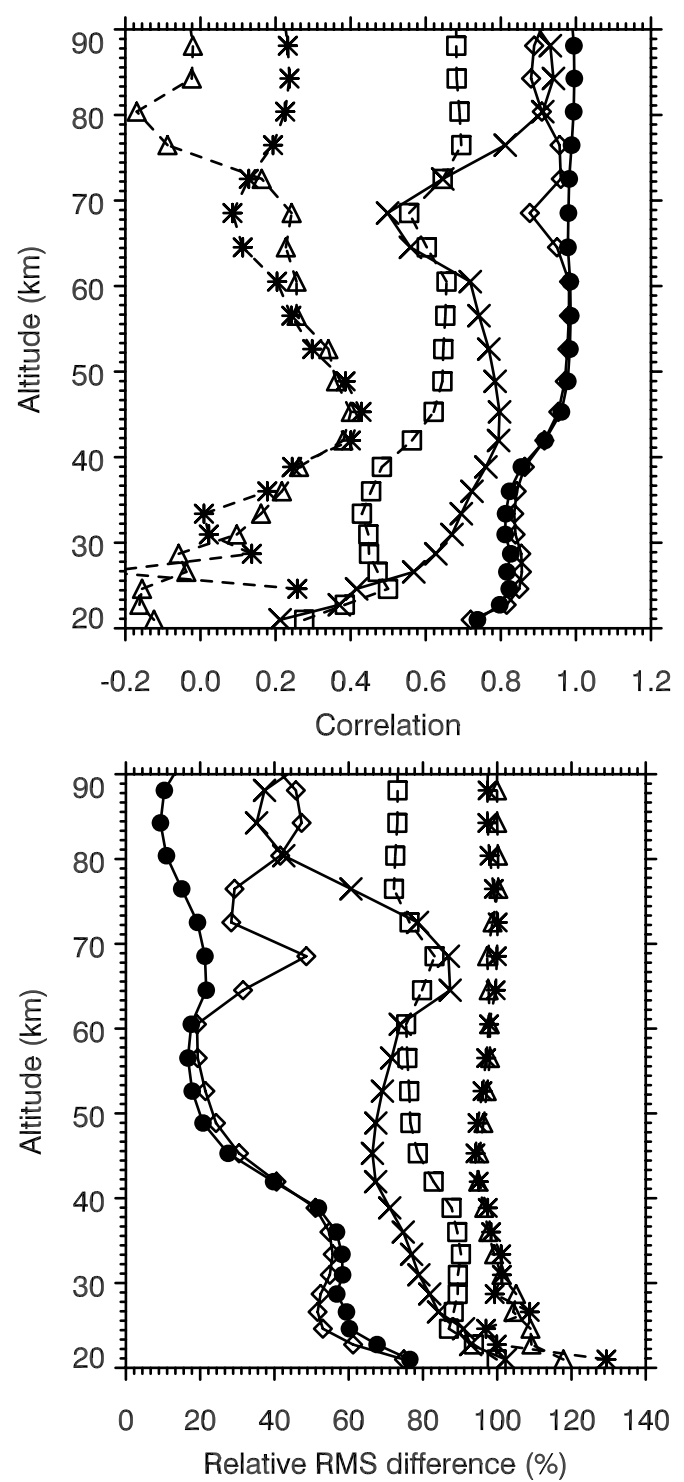

\section{SOLSTICE}
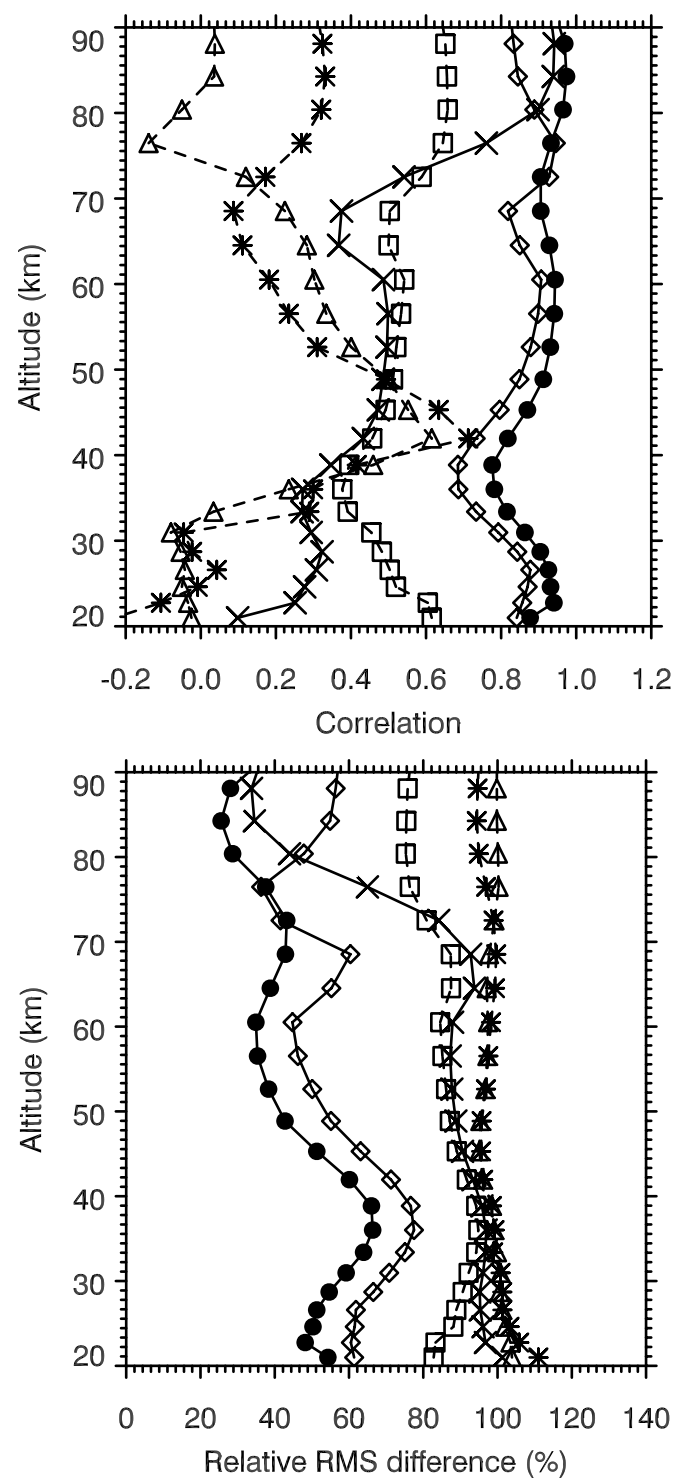

Fig. 5. Temperature: Vertical profiles of the correlation (upper row) and relative RMS difference (lower row) of daily variations of the temperature for SUSIM (left column) and SOLSTICE (right column) with different proxies: Ly- $\alpha$ (solid line, crosses), $205 \mathrm{~nm}$ (solid line, diamonds), LYRA-P (solid line, filled circles), TSI (dashed line, triangles), F10.7(dashed line, squares), and NMD (dashed line, asterisks).

middle atmosphere state above $40 \mathrm{~km}$. Some problem with ozone simulation between 40 and $70 \mathrm{~km}$ has been encountered when the SOLSTICE solar irradiance is used as the reference. This problem is caused by the enhanced variability of the SOLSTICE solar irradiance in the ozone Hartley band. At the moment it is hard to say whether this problem is real or it is just instrumental error. We would like to remind the reader that the SUSIM and SOLSTICE observations are not purely simultaneous but still refer to the state of the Sun of the same day. Thus, the difference between SUSIM and SOLSTICE data is an indication of the reliability of the observations. Probably, further study with different reference solar irradiance data set (SORCE, Rottman et al., 2006) will help to solve this problem. Lower accuracy has been obtained using Ly- $\alpha$, HERZ and $205 \mathrm{~nm}$ proxies. Ly- $\alpha$ proxy can be successfully used in the mesosphere and HERZ and $205 \mathrm{~nm}$ proxies give good accuracy in the stratosphere, but the accuracy of the combined LYRA-P proxy is superior. The solar irradiance reconstructed from F10.7, TSI and NMD proxies does not provide satisfactory results. It is important to emphasize that all conclusions presented here are valid for daily time scale. The correlation between solar irradiance and considered proxies can be rather different for hourly, monthly and annual time scales. 
Acknowledgements. This work has been supported by Swiss National Science Foundation (grant N 200020-101848/1) and Swiss COST-724 (grant C04.0106). We thank N. Krivova and one anonymous reviewer for their helpful comments and suggestions.

Edited by: M. Blumthaler

\section{References}

Brasseur, G. and Solomon, S.: Aeronomy of the middle atmosphere: Chemistry and physics of the stratosphere and mesosphere, Third edition, Springer, Dordrecht, the Netherlands, 646 pp., 2005.

Dudok de Wit, T., Lilensten, J., Aboudarham, J., Amblard, P.-O., and Kretzschmar, M.: Retrieving the solar EUV spectrum from a reduced set of spectral lines, Ann. Geophys., 23, 3055-3069, 2005, http://www.ann-geophys.net/23/3055/2005/.

Egorova, T. A., Karol, I. L. and Rozanov, E. V.: The influence of ozone content loss in the lower stratosphere on the radiative balance of the troposphere, Physics of atmosphere and ocean (Russian Academy of Sciences), 33, N4, 492-499, 1997.

Floyd, L. E., Reiser, P. A., Crane, P. C., Herring, L. C., Prinz, D. K., Brueckner, G. E.: Solar cycle 22 UV spectral irradiance variability: current measurements by SUSIM UARS, Solar Phys., 177, 79-87, 1998.

Fomichev, V. I., Blanchet, J.-P., and Tuner, D. S.: Matrix parameterization of the 15 micro $\mathrm{CO} 2$ band cooling in the middle and upper atmosphere for variable CO2 concentration, J. Geophys. Res., 103, 11 505-11 528, 1998.

Frolkis, V. A. and Rozanov, E. V.: Radiation code for climate and general circulation models. IRS'92: Current problems in atmospheric radiation, Tallin, Estonia, 3-8 August, 176-179, 1992.

Jansen, F, Pirjola, R., and Favre, R.: Space weather: Hazard to the Earth?, Swiss Re Publishing, Zurich, 39 pp., 2000.

Heaps, M. G.: Parameterization of the cosmic ray ion-pair production rate above $18 \mathrm{~km}$, Planet Space Sci., 26, 513-517, 1978.

Hochedez, J.-F., Schmutz, W., Stockman, Y., et al.: LYRA: the Solar UV radiometer aboard the ESA Proba-2, LYRA, a solar UV radiometer on Proba2, Adv. Space Res., 37, 303-312, 2006.

Kazil, J.: The University of Bern Atmospheric Ion Model: TimeDependent Ion Modeling in the Stratosphere, Mesosphere and Lower Thermosphere, PhD Thesis, University of Bern, Switzertland, 2002.
Koop, E.: Electron and ion dencities, in: The Upper Atmosphere, edited by: W. Dieminger, G. K., Hartman, R. leitinger, Springer, 1996.

Krivova, N. A, Solanki, S. K., and Floyd, L.: Reconstruction of solar UV irradiance in cycle 23, Astronomy and Astrophysics, 452, 631-639, 2006.

Rottman, G. J., Woods, T. N., and Sparn, T. P.: Solar stellar irradiance comparison experiment I: 1 instrument design and operation, J. Geophys. Res. 98, $10667-10677,1993$.

Rottman, G., Woods, T., and McClintock, W.: SORCE solar UV irradiance results, Adv. Space Res., 37, 2, 201-208, 2006.

Rozanov, E. V., Zubov, V. A., Schlesinger, M. E., Yang, F., and Andronova N. G.: The UIUC 3-D Stratospheric Chemical Transport Model: Description and Evaluation of the simulated Source Gases and Ozone, J. Geophys. Res., 104, 11 755-11 781, 1999.

Rozanov, E., Egorova T., Fröhlich, C., Haberreiter, M., Peter, T., and Schmutz, W.: Estimation of the Ozone and Temperature Sensitivity to the Variation of Spectral Solar Flux, in: SOHO 11 Symposium From Solar Min to Max: Half a Solar Cycle with SOHO, edited by: A. Wilson, ESA SP-508, ESA Publications Division, Noordwijk, The Netherlands, 181-184, 2002.

Rozanov, E., Egorova, T., and Schmutz, W.: Response of the Earth's Atmosphere to the Solar Irradiance Variability, in: Climate Variability and Extremes during the Past 100 Years, edited by: Brönnimann, S., Luterbacher, J., Ewen, T., Diaz, H. F., Stolarski, R. S., and Neu, U., Advances in Global Change Research, 33, 317-331, Springer, 2008.

Sander, S. P., Friedl, R., Golden, D., et al.: Chemical kinetics and photochemical data for use in atmospheric studies: Evaluation number 14, Tech. Rep. JPL Publ. 02-25, Jet Propulsion Laboratory, 2003.

Thuillier, G., Dewitte, S., Schmutz, W., and the Picard Team: Simultaneous measurement of the total solar irradiance and solar diameter by the PICARD mission, Adv. Space Res., 38, 1792 1806, 2006.

Unruh, Y., Krivova, N., Solanki, S., Harder, J., and Kopp, G.: Spectral irradiance variations: Comparison between observations and the SATIRE model on solar rotation time scales, Astronomy and Astrophysics, accepted, 2008.

Wilkinson, D. C.: National Oceanic and Atmospheric Administration's spacecraft anomaly data base and examples of solar activity affecting spacecraft, J. Spacecraft and Rockets, 31, MarchApril, 1994.

Woods, T. N., Prinz, D. K., Rottman, G. J., et al.: Validation of the UARS solar ultraviolet irradiances: Comparison with ATLAS 1 and 2 measurements, J. Geophys. Res., 101, 9541-9569, 1996. 